\title{
Laryngotracheoplastic resection for primary tumors of the proximal airway
}

\author{
Henning A. Gaissert, MD, a,b Hermes C. Grillo, MD, ${ }^{a}$ Behgam M. Shadmehr, MD, ${ }^{a}$ Cameron D. Wright, MD, \\ Manjusha Gokhale, MA, ${ }^{c}$ John C. Wain, MD, and Douglas J. Mathisen, MD ${ }^{a}$
}

Background: Primary tumors of the airway with proximity to vocal cords and recurrent laryngeal nerves can be resected with sparing of the larynx. Long-term data on survival and local recurrence after laryngotracheal resection are scarce.

Methods: We conducted a retrospective study of laryngotracheal resection and reconstruction for primary tumors of the airway since 1972.

Results: Twenty-five patients aged 15 to 77 years presented with adenoid cystic carcinomas $(n=9)$, squamous cell carcinomas $(n=6)$, and other airway tumors $(\mathrm{n}=10)$. Subglottic resection consisted of anterior cricoid in 5 patients; posterior cricoid mucosa in 9 patients, with resection of the posterior cricoid plate in 3 patients; lateral resection in 7 patients; and combined anterior and posterior elements in 4 patients. Vascularized trachea was tailored to reconstruct the defect. Seven patients without hoarseness required resection of the recurrent laryngeal nerve, and 4 other patients with hoarseness did not. There were no operative deaths. Two $(8.0 \%)$ patients who had received prior high-dose cervical radiation had anastomotic separation, one requiring laryngectomy. One patient needed permanent tracheostomy, and temporary ( $<2$ months) airway tubes were used in 5 patients. Sixteen patients received postoperative radiation. Median follow-up was 101 months. Four (16\%) patients died of disease. Overall survival at 5 and 10 years was $79 \%$ and $64 \%$, respectively. No patient underwent laryngectomy for recurrence.

Conclusion: Laryngotracheal resection and immediate reconstruction for subglottic tumors is achieved with good preservation of voice, low morbidity, and no compromise of long-term survival.

From the Division of Thoracic Surgery ${ }^{\mathrm{a}}$ and the Center for Clinical Effectiveness in Surgery, ${ }^{\mathrm{b}}$ Massachusetts General Hospital and Harvard Medical School, Boston, Mass, and Policy Analysis, Inc, ${ }^{\mathrm{c}}$ Brookline, Mass.

Received for publication May 4, 2004; revisions received July 6, 2004; accepted for publication July 13, 2004.

Address for reprints: Henning A. Gaissert, MD, Massachusetts General Hospital, Blake 1570, Fruit Street, Boston, MA 02114 ( E-mail: hgaissert@partners.org).

J Thorac Cardiovasc Surg 2005;129:1006-9

$0022-5223 / \$ 30.00$

Copyright $\odot 2005$ by The American Association for Thoracic Surgery

doi:10.1016/j.jtcvs.2004.07.043
$\mathrm{S}$ ome primary neoplasms of the airway arise in the subglottic larynx or extend upward from the cervical trachea. A larynx-conserving procedure deliberately compromises the extent of resection in these tumors but is preferable to preserve vocal cord function as long as local recurrence does not affect long-term survival and close margins are controlled by radiation. Pearson and associates ${ }^{1}$ found no local recurrence among 7 patients with various tumors from 1 to 12 years after subglottic resection. There are few other data on long-term follow-up.

Two technical alternatives exist: tumor excision by means of staged reconstruction with cartilage or soft tissue patches or single-stage circumferential resection by means of thyrotracheal anastomosis. We prefer the latter technique, previously reported for postintubation stenosis, ${ }^{2-4}$ idiopathic strictures, ${ }^{5}$ and thyroid carcinoma, ${ }^{6}$ because the vascularized tracheal wall creates immediate stability of the airway so that most patients require neither temporary tracheostomy nor multiple operations. This study describes the procedures, early result, and long-term follow-up after laryngotracheal resection for primary airway tumors. 


\section{Methods}

Hospital, office, and pathology records at the Massachusetts General Hospital (MGH) were searched for patients undergoing laryngotracheoplastic resection for primary airway tumors. Patients were contacted, and the Social Security Death Index was searched. Follow-up was complete; the cause of death was unknown in 1 patient. The MGH Human Research Committee permitted the study, and patients provided consent for follow-up.

Tumor extension to the vocal cords at endoscopy was a contraindication to larynx-conserving resection. Sacrifice of one recurrent laryngeal nerve was accepted when tumor encased the nerve and the contralateral nerve was preserved. Patients who required simple beveling of a margin of lower cricoid cartilage were excluded because reconstruction demands only end-to-end cricotracheal anastomosis. Anterior tumors were removed by means of cricoid excision, dividing the lateral arches as far back as the posterior cricoid plate and high enough to incorporate the cricothyroid membrane or the lower thyroid cartilage. Extensive involvement of the lateral subglottic wall and ipsilateral recurrent nerve required removal of up to half of the cricoid with part of the posterior cricoid plate and the thyroid lamina. Tumor attachment to the posterior cricoid plate alone was removed by means of sloping excision of the mucosa with or without tangential excision of the posterior cartilage. Division of the anterior cricoid (cricofissure) improved access to the posterior cricoid plate. Surgical techniques of laryngotracheal resection have been previously described., ${ }^{3,9}$

Resection after previous high-dose radiation of the neck was undertaken with substernal advancement of pedicled omentum to augment local blood supply and to buttress the laryngotracheal anastomosis. ${ }^{10}$ The omentum was wrapped around the entire anastomosis and sutured to the larynx and trachea above and below the anastomosis. ${ }^{9}$ Patients with malignant tumors and close (within a few millimeters) or microscopically positive resection margins underwent adjuvant radiotherapy (5400-6000 cGy) 4 to 6 weeks after the operation. Bronchoscopic assessment of the anastomosis preceded radiation treatment.

\section{Results}

Of 341 patients with primary airway tumors between 1972 and 2002, 25 patients (15 men) with a mean age of 51 years (range, 15-77 years) underwent laryngotracheoplastic resection. Nine patients underwent laryngectomy at $\mathrm{MGH}$ for a primary tracheal tumor in this interval (unpublished data). Table 1 lists tumor histology. The mean duration of symptoms was 12 months (range, 1-60 months). Symptoms related to airway obstruction dominated (Table 2). Hemoptysis was present in 5 of 6 patients with squamous cell carcinoma but in none of 8 patients with adenoid cystic carcinoma. Five patients had prior tumors: 3 had had vocal cord carcinoma radiated with doses of greater than 6000 cGy, 1 had a thyroid carcinoma, and 1 had a carcinoma in situ of the cervix. Oral corticosteroids were discontinued before resection in 3 patients.

Four patients had tracheostomy before referral to $\mathrm{MGH}$, and 1 was transferred intubated. Local tumor was removed before resection in 9 patients by means of core-out in 6
TABLE 1. Tumor histology

\begin{tabular}{lc}
\hline Type of tumor & No. of patients \\
\hline Total & 25 \\
Adenoid cystic carcinoma & 9 \\
Squamous cell carcinoma & 6 \\
Mucoepidermoid carcinoma & 2 \\
Spindle cell sarcoma & 2 \\
Chondrosarcoma & 2 \\
Paraganglioma & 1 \\
Granular cell tumor & 1 \\
Plasma cell granuloma & 1 \\
Lymphoma & 1 \\
\hline
\end{tabular}

TABLE 2. Presenting symptoms

\begin{tabular}{lrr}
\hline Symptoms and signs & No. & $\%$ \\
\hline Dyspnea & 18 & 72 \\
Stridor & 11 & 44 \\
Cough & 8 & 32 \\
Wheeze & 7 & 28 \\
Hemoptysis & 6 & 24 \\
Hoarseness & 4 & 16 \\
Dysphagia & 3 & 12 \\
Pain & 1 & 4
\end{tabular}

patients, laser in 2 patients, and photodynamic therapy in 1 patient. An endoscopic search for synchronous airway tumors located a second squamous cell carcinoma in the lower trachea in 1 patient and a second granular cell tumor in the bronchus intermedius in another patient.

\section{Resection and Reconstruction}

Resection of the subglottic space was divided into 4 types: resection of the anterior or anterolateral cricoid in 5 patients; resection of the posterior cricoid mucosa in 9 patients, with tangential excision of the cricoid plate in 3 patients; lateral resection in 7 patients; and combined anterior and posterior elements in 4 patients. The length of the resected trachea measured 0.5 to $4.6 \mathrm{~cm}$ (mean, 2.9 $\mathrm{cm})$. A recurrent nerve involved by tumor was resected in 7 patients, of whom 6 had adenoid cystic carcinoma and 1 had plasma cell granuloma. An involved nerve was sacrificed in 4 of 6 patients during lateral laryngeal resection; the remaining 2 lateral resections for chondrosarcoma could be conducted in a subperichondrial plane. Omentum was used to cover the laryngotracheal anastomosis in 3 patients with previous radiation of the neck. No anastomosis was stented with a tracheal $\mathrm{T}$ tube at the time of resection.

Table 3 details the depth of tumor invasion, concomitant resection, margins, and lymph node status. Although "pos- 
TABLE 3. Tumor characteristics by tumor type

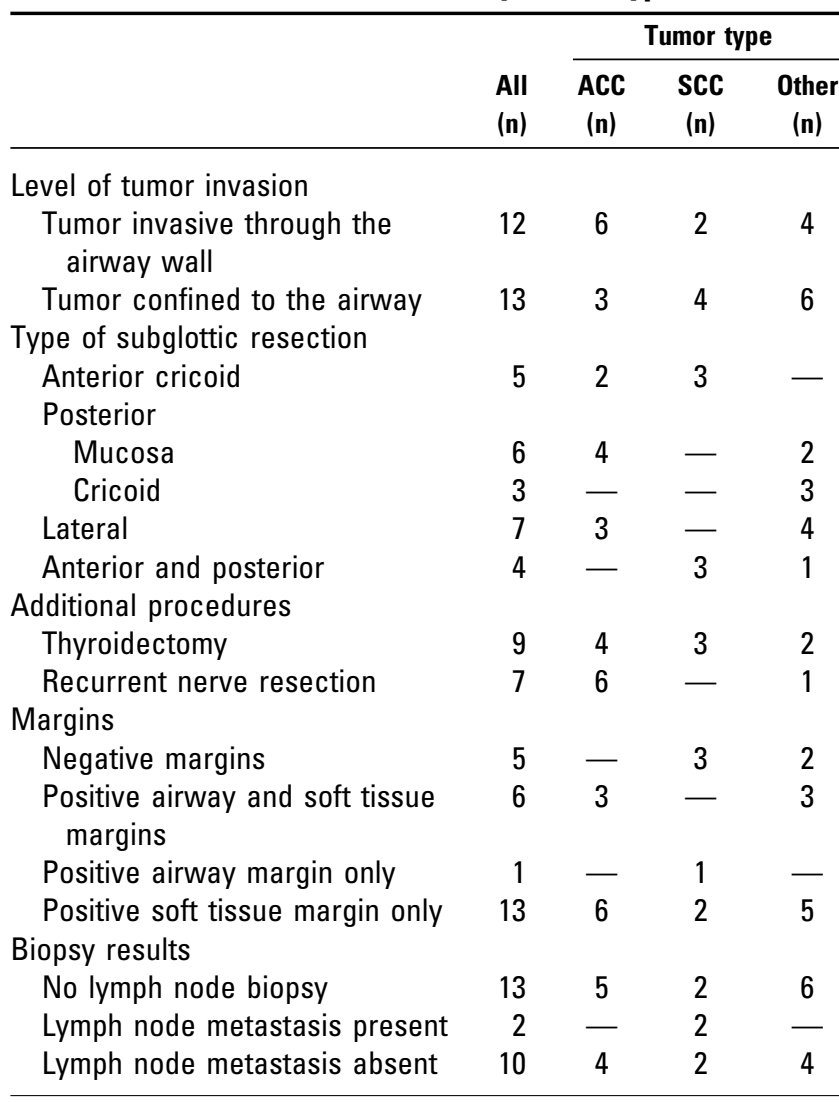

ACC, Adenoid cystic carcinoma; SCC, squamous cell carcinoma.

itive airway margin" indicates microscopic tumor found at the resection line, "positive soft tissue margin" is determined at the lateral limit of resection of the specimen and does not necessarily indicate the presence of residual tumor cells. Four patients had local resection of esophageal muscle involved with tumor. Patients with adenoid cystic carcinoma were more likely to have invasion through the wall, positive margins, and resection of the recurrent nerve than those with squamous cell carcinoma. ${ }^{11}$

\section{Operative Results}

There were no operative deaths. Seventeen patients had an uneventful postoperative recovery and breathed with unassisted airway. Two patients with prior neck radiation had anastomotic separation with cervical abscesses contained by the omentum, which had failed to promote healing. One was managed with a temporary $\mathrm{T}$ tube, but the other required laryngectomy. Another patient experienced bilateral vocal cord paralysis after circumferential subglottic and unilateral recurrent nerve resection. She has a tracheostomy and is awaiting a procedure to enlarge the glottic opening. Five additional patients, 3 after lateral laryngeal and recurrent nerve resection and 2 after posterior cricoid resection with mucosal flap reconstruction, needed temporary tracheos-
TABLE 4. Survival by tumor type

\begin{tabular}{lrrrr}
\hline & & \multicolumn{3}{c}{ Tumor type } \\
\cline { 3 - 5 } & All & ACC & SCC & Other \\
\hline No. of patients & 25 & 9 & 6 & 10 \\
Survivals (\%) & & & & \\
$\quad$ Survived the operation & 100 & 100 & 100 & 100 \\
1 y & 92 & 100 & 83 & 100 \\
5 y & 79 & 100 & 60 & 100 \\
$10 y$ & 64 & 80 & 50 & 100 \\
\hline
\end{tabular}

ACC, Adenoid cystic carcinoma; SCC, squamous cell carcinoma.

tomy tubes $(\mathrm{n}=3)$ or insertion of a temporary $\mathrm{T}$ tube $(\mathrm{n}=$ 2). All 5 were decannulated in less than 2 months and are now breathing without difficulty. Thus $6(24 \%)$ patients required temporary airway tubes, and $1(4.0 \%)$ patient required a permanent tube.

\section{Postoperative Treatment}

Postoperative radiotherapy ranging from 5000 to $6300 \mathrm{cGy}$ was given to 16 patients. This group included all 9 patients with adenoid cystic carcinoma, 3 with squamous cell carcinoma, and 1 each with mucoepidermoid carcinoma, spindle cell sarcoma, chondrosarcoma, and lymphoma.

\section{Long-Term Results}

The median follow-up period is 101 months (range, 5-359 months). Survivals are given in Table 4. Seventeen patients have no evidence of disease. Three are alive with disease: one with distant recurrence of squamous cell carcinoma, another with locally recurrent chondrosarcoma, and one with lymphoma. Three patients with adenoid cystic carcinoma died of distant disease; one of them had undergone resection of the same tumor in the right main stem bronchus 11 years after laryngotracheal resection, which was thought to be a regional recurrence. Two patients with squamous cell carcinoma died 5 and 34 months after resection, one of distant disease and the other of unknown cause.

\section{Discussion}

We first applied laryngotracheal resection to postintubation injuries and other benign conditions. ${ }^{3}$ Compared with tumors, these strictures are poorly defined in extent and often require the surgeon to suture to a somewhat diseased subglottic larynx. In neoplastic disease, however, the normal and stable airway wall is used for reconstruction. One-stage laryngotracheal resection of primary airway tumors hence usually resulted in a dependable airway. The rate of local recurrence, despite positive soft tissue or airway margins in $78 \%$ of patients, was surprisingly low. Most patients did not require further surgical procedures, although they are advised of a potential need for later laryngectomy. When death from disease occurred, it was due to distant metastasis. In 
selected patients laryngotracheal resection is therefore preferable to laryngectomy. Despite the low incidence of subglottic cancers, ${ }^{12}$ the operation seems underused. This experience parallels that with extrinsic tumors, namely thyroid carcinoma with airway invasion. ${ }^{6}$

Whether laryngeal function should be sacrificed if a microscopic tumor is found on an intraoperative frozen section in a case in which additional local resection is not feasible is a matter of judgment and tumor type for an individual patient. Control of microscopic disease in adenoid cystic disease seems possible in some cases. ${ }^{11}$ The risk incurred for survival is unknown. In the rare case of lowgrade chondrosarcoma, which typically arises from the cricoid cartilage, larynx-sparing resection aims at prolonged palliation of vocal cord function, reserving completion laryngectomy for a distant future.

Staged laryngotracheal reconstruction offers no advantage over immediate anastomosis. Staging commonly requires tracheostomy, prolonged internal stenting, and multiple surgical procedures. Pedicled composite grafts, for example, require at least 3 months from the first operation to decannulation and frequently use synthetic material. ${ }^{13}$ The obligatory 2 or 3 operative stages do not always result in an unsupported airway. For benign indications, composite nasal grafts did not allow decannulation in $31 \%$ of patients, and airway tubes were required for a mean of 3.7 months in the others. ${ }^{14}$ Staged skin flap advancement for intubation strictures failed in $24 \%$, with 3 to 8 surgical procedures in patients who were eventually decannulated. ${ }^{15}$ The trachea forms an excellent subglottic replacement when long airway segments (up to 4.6 $\mathrm{cm}$ in this group) are resected and could potentially cover even hemilaryngectomy defects.

\section{References}

1. Pearson FG, Brito-Filomeno L, Cooper JD. Experience with partial cricoid resection and thyrotracheal anastomosis. Ann Otol Rhinol Laryngol. 1986;95:582-5.

2. Pearson FG, Cooper JD, Nelems JM, Van Nostrand AW. Primary tracheal anastomosis after resection of the cricoid cartilage with preservation of recurrent laryngeal nerves. J Thorac Cardiovasc Surg. 1975;70:806-16.

3. Grillo HC. Primary reconstruction of airway after resection of subglottic laryngeal and upper tracheal stenosis. Ann Thorac Surg. 1982; 33:3-18.

4. Couraud L, Brichon PY, Velly JF. The surgical treatment of inflammatory and fibrous laryngotracheal stenosis. Eur J Cardiothorac Surg. 1988;2:410-5.

5. Grillo HC, Mark EJ, Mathisen DJ, Wain JC. Idiopathic laryngotracheal stenosis and its management. Ann Thorac Surg. 1993;56:80-7.

6. Grillo HC, Suen HC, Mathisen DJ, Wain JC. Resectional management of thyroid carcinoma invading the airway. Ann Thorac Surg. 1992;54: 3-9.

7. Grillo HC, Mathisen DJ, Wain JC. Laryngotracheal resection and reconstruction for subglottic stenosis. Ann Thorac Surg. 1992;53: 54-63.

8. Grillo HC, Zannini P. Resectional management of airway invasion by thyroid carcinoma. Ann Thorac Surg. 1986;42:287-98.

9. Grillo HC. Surgery of the trachea and bronchi. Hamilton (Canada): Decker; 2003.

10. Muehrcke DD, Grillo HC, Mathisen DJ. Reconstructive airway operation after irradiation. Ann Thorac Surg. 1995;59:14-8.

11. Gaissert HA, Grillo HC, Shadmehr MB, Wright CD, Gokhale M, Wain JC, et al. Long-term survival after resection of primary adenoid cystic and squamous cell carcinoma of the trachea and carina. Ann Thorac Surg. 2004;78:1889-97.

12. Harrison DF. The pathology and management of subglottic cancer. Ann Otol Rhinol Laryngol. 1971;80:6-12.

13. Lindholm CE, Lofgren L. Airway repair with pedicled composite grafts-clinical experience. Otolaryngol Head Neck Surg. 1987;96: 48-54.

14. Duncavage JA, Ossoff RH, Toohill RJ. Laryngotracheal reconstruction with composite nasal septal cartilage grafts. Ann Otol Rhinol Laryngol. 1989;98:581-5.

15. Biller HF, Lawson W, Weisberg V. Staged repair of extensive tracheal and laryngotracheal stenoses. Ann Otol Rhinol Laryngol. 1986;95: 586-9. 